\title{
Modelling of Stagnation-Point Flow and Diffusion of Chemically Reactive Species Past A Permeable Quadratically Stretching/Shrinking Sheet
}

\author{
Roslinda Nazar* \\ School of Mathematical Sciences, Faculty of Science \& \\ Technology \\ Universiti Kebangsaan Malaysia \\ 43600 UKM Bangi, Selangor, Malaysia \\ *Corresponding author \\ Ezad Hafidz Hafidzuddin \\ School of Mathematical Sciences, Faculty of Science \& \\ Technology \\ Universiti Kebangsaan Malaysia \\ 43600 UKM Bangi, Selangor, Malaysia
}

\author{
Norihan Md Arifin \\ Department of Mathematics \\ Universiti Putra Malaysia \\ 43400 UPM Serdang, Selangor, Malaysia
}

\author{
Ioan Pop
Department of Mathematics
Babes-Bolyai University
400084 Cluj-Napoca, Romania \\ Ioan Pop
Department of Mathematics
Babes-Bolyai University
400084 Cluj-Napoca, Romania \\ Ioan Pop
Department of Mathematics
Babes-Bolyai University
400084 Cluj-Napoca, Romania \\ Ioan Pop
Department of Mathematics
Babes-Bolyai University
400084 Cluj-Napoca, Romania
}


analytical solution for the problem of flow and diffusion of chemically reactive species over a nonlinearly stretching sheet immersed in a porous medium, while Bhattacharyya [34] obtained dual solutions for the problem of boundary layer stagnation-point flow and mass transfer with chemical reaction past a stretching/shrinking sheet. Roşca et al. [35] investigated the steady forced convection stagnation-point flow and mass transfer past a permeable stretching/shrinking sheet in a nanofluid. The steady two-dimensional MHD stagnation-point flow towards a permeable stretching sheet with chemical reaction was studied by Rasekh et al. [36]. Recently, the unsteady two-dimensional boundary layer stagnation-point flow of a nanofluid over a heated stretching sheet is investigated numerically by Abd El-Aziz [37], while Najib et al. [38] discussed the stagnation-point flow and mass transfer with the chemical reaction past a stretching/shrinking cylinder.

The aim of the present study is to extend the work done by Ziabakhsh et al. [33] by considering the stagnation-point flow past a permeable quadratically stretching/shrinking sheet. The governing partial differential equations are reduced to a system of nonlinear ordinary differential equations by using similarity transformations and solved numerically. The effects of the governing parameters on the skin friction coefficient and concentration gradient are investigated and discussed.

\section{GOVERNING EQUATIONS}

Consider the steady two-dimensional stagnation-point flow and diffusion of chemically reactive species of a viscous and incompressible fluid over a permeable quadratically stretching/ shrinking sheet, where $x$ and $y$ are the Cartesian coordinates measured along and normal to the surface of the sheet, respectively. It is assumed that the surface is stretched/shrunk in the $x$-direction with the velocity $u_{w}(x)$, the mass transfer velocity is $v_{w}(x)$ and the velocity of the far flow (inviscid flow) is $u_{e}(x)$.

$$
\begin{gathered}
\frac{\partial u}{\partial x}+\frac{\partial v}{\partial y}=0 \\
u \frac{\partial u}{\partial x}+v \frac{\partial u}{\partial y}=u_{e} \frac{d u_{e}}{d x}+v \frac{\partial^{2} u}{\partial y^{2}} \\
u \frac{\partial C}{\partial x}+v \frac{\partial C}{\partial y}=D \frac{\partial^{2} C}{\partial y^{2}}-k_{1} C
\end{gathered}
$$

We shall solve (1)-(3) subject to the following boundary conditions:

$$
\left.\begin{array}{l}
u=u_{w}(x)=a \lambda x+b \lambda x^{2}, \\
v=v_{w}(x)=v_{0}+v_{0} \frac{x}{c}, C=C_{w},
\end{array}\right\} \text { at } y=0,
$$

where $u$ and $v$ are the velocity components along the $x$ and $y$ axes, respectively, $C$ is the species concentration in the fluid, $v$ is the kinematic viscosity, $k_{1}$ is the chemical reaction rate, $D$ is the mass diffusion coefficient, $a, b$ and $c$ are positive constants with $c(\neq 0), v_{0}$ is the constant mass velocity with $v_{0}<0$ for suction and $v_{0}>0$ for injection, and $\lambda$ is the constant stretching/shrinking parameter, with $\lambda>0$ for a stretching sheet and $\lambda<0$ for a shrinking sheet, respectively. It is assumed that the diffusing species is destroyed, i.e. destructive chemical reaction $k_{1}>0$ in the homogeneous reaction. We notice that when $b=0$, it corresponds to the linearly stretching/shrinking sheet. The other case is $b \neq 0$, which corresponds to a quadratic stretching/shrinking sheet.

Introducing the following similarity solutions (see [33])

$$
\begin{aligned}
& u=a x f^{\prime}(\eta)+b x^{2} g^{\prime}(\eta), v=-\sqrt{a v} f(\eta)-\frac{2 x b}{\sqrt{a / v}} g(\eta), \\
& C=C_{w}\left[F(\eta)+\frac{2 b x}{a} h(\eta)\right], \eta=\sqrt{\frac{a}{v}} y
\end{aligned}
$$

where primes denote differentiation with respect to $\eta$.

Substituting (5) into (2) and (3), the following set of ordinary differential equations results in

$$
\begin{gathered}
f^{\prime \prime \prime}+f f^{\prime \prime}+1-f^{\prime 2}=0, \\
g^{\prime \prime \prime}+f g^{\prime \prime}-3 f^{\prime} g^{\prime}+2 f^{\prime \prime} g+3=0, \\
F^{\prime \prime}+S c f F^{\prime}-K F=0, \\
h^{\prime \prime}+S c\left(f h^{\prime}-f^{\prime} h+F^{\prime} g\right)-K h=0,
\end{gathered}
$$

subject to the boundary condition

$$
\begin{aligned}
& f(0)=S, f^{\prime}(0)=\lambda, g(0)=S_{1}, g^{\prime}(0)=\lambda, F(0)=1, h(0)=0 \\
& f^{\prime}(\eta) \rightarrow 1, g^{\prime}(\eta) \rightarrow 1, F(\eta) \rightarrow 0, h(\eta) \rightarrow 0 \text { as } \eta \rightarrow \infty
\end{aligned}
$$

where $S c$ is the Schmidt number, $K$ is the dimensionless homogeneous reaction parameter and $S$ and $S_{1}$ (where $S=S_{1}$ ) are the dimensionless velocity mass flux parameters with $\left(S, S_{1}\right)>0$ for suction and $\left(S, S_{1}\right)<0$ for injection, which are defined as

$$
S c=\frac{v}{D}, K=\frac{k_{1} S c}{a}, S=-\frac{v_{0}}{\sqrt{a v}}, S_{1}=-\frac{v_{0}}{2 b c} \sqrt{\frac{a}{v}} .
$$

The quantities of physical interest are the skin friction coefficient $C_{f}$ and the local Sherwood number $S h_{x}$, which are defined as

$$
C_{f}=\frac{\tau_{w}}{\rho(a x)^{2}}, S h_{x}=\frac{x q_{m}}{D C_{w}},
$$


where $\tau_{w}$ is the skin friction or the shear stress along the surface, $\rho$ is the fluid density and $q_{m}$ is the surface concentration of the reactant, which are given by

$$
\tau_{w}=\mu\left(\frac{\partial u}{\partial y}\right)_{y=0}, q_{m}=-D\left(\frac{\partial C}{\partial y}\right)_{y=0} .
$$

Using (5) into (12) and (13), we obtain

$$
\begin{aligned}
& \operatorname{Re}_{x}^{1 / 2} C_{f}=f^{\prime \prime}(0)+\frac{b}{a} x g^{\prime \prime}(0), \\
& \operatorname{Re}_{x}^{-1 / 2} S h_{x}=-F^{\prime}(0)-\frac{2 b x}{a} h^{\prime}(0),
\end{aligned}
$$

where $\operatorname{Re}_{x}=(a x) x / v$ is the local Reynolds number.

\section{RESULTS AND DISCUSSION}

The nonlinear ordinary differential equations (6)-(9) with boundary conditions (10) are solved numerically by using the "bvp4c" function from MATLAB (see Kierzenka and Shampine [39]). To validate the accuracy of the present numerical method, the values of the reduced skin friction coefficient $f^{\prime \prime}(0)$ are compared with [34], which can be observed in Table 1. The comparisons are found to be in excellent agreement, and thus we are confident that the present method is accurate.

Figure 1 displays the variations of the reduced skin friction coefficients $f^{\prime \prime}(0)$ with $\lambda$ for some values of $S$ when $K=0.5$ and $S c=0.6$. The values of $f^{\prime \prime}(0)$ are seen to increase with the increase of $S$. It can be seen that there exists more than one solution for a certain range of the stretching/shrinking parameter $\lambda$. The dual solutions are obtained by setting two different initial guesses for the missing values of $f^{\prime \prime}(0), g^{\prime \prime}(0)$, $-F^{\prime}(0)$ and $-h^{\prime}(0)$, where all profiles satisfy the far field boundary conditions (10) asymptotically. It appears that the solution is unique for $\lambda>-1$, while the dual solutions exist for $\lambda_{c} \leq \lambda \leq-1$, and no solution for $\lambda<\lambda_{c}$, where $\lambda_{c}$ is the critical values of $\lambda$. Beyond these critical points, the boundary layer separates from the surface, hence the solutions based upon the boundary layer approximations are not possible. Figure 1 also shows that the values of $\left|\lambda_{c}\right|$ increase with the increase of $S$. This indicates that mass flux parameter $S$ widen the range of $\lambda$ for which solutions exist.

TABLE I. COMPARISON OF THE VALUES OF $f^{\prime \prime}(0)$ WITH [34] FOR SOME VALUES OF ${ }^{\lambda}$ WHEN $S=0$

\begin{tabular}{lcccc}
\hline & \multicolumn{2}{c}{$[34]$} & \multicolumn{2}{c}{ Present results } \\
\cline { 2 - 5 }$\lambda$ & $\begin{array}{c}\text { First } \\
\text { solution }\end{array}$ & $\begin{array}{c}\text { Second } \\
\text { solution }\end{array}$ & $\begin{array}{c}\text { First } \\
\text { solution }\end{array}$ & $\begin{array}{c}\text { Second } \\
\text { solution }\end{array}$ \\
\hline-1 & 1.3288169 & 0 & 1.3288169 & 0 \\
-1.2 & 0.9324728 & 0.2336491 & 0.9324734 & 0.2336497 \\
-1.2465 & 0.5842915 & 0.5542856 & 0.5842979 & 0.5542962 \\
-1.24657 & 0.5745268 & 0.5639987 & 0.5745599 & 0.5640126 \\
\hline
\end{tabular}

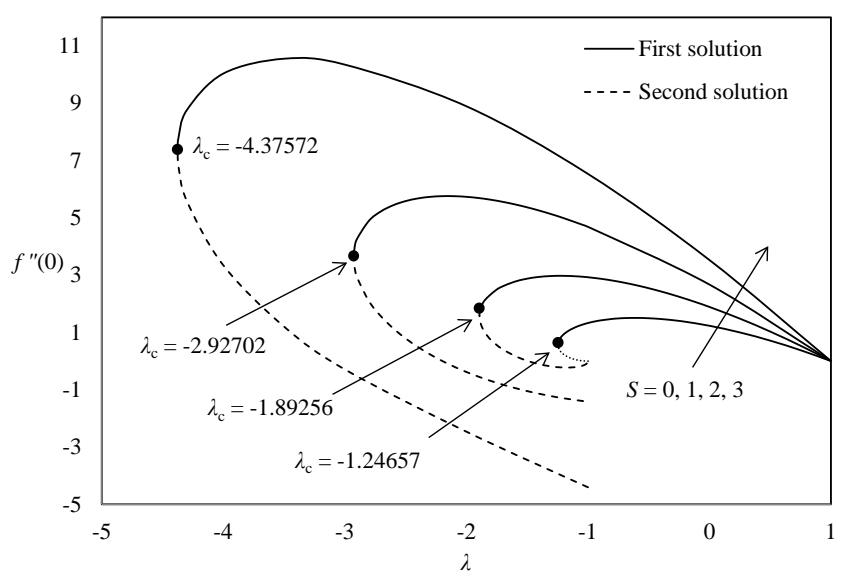

FIGURE I. VARIATIONS OF $f^{\prime \prime}(0)$ WITH $\lambda$ FOR DIFFERENT S WHEN $K=0.5, S c=0.6$

Figure 2 illustrates the variations of the concentration gradients $-h^{\prime}(0)$ with $\lambda$ for various values of reaction parameter $K$ when $S=3$ and $S c=0.6$. It can be observed that the values of $-h^{\prime}(0)$ are decreasing with the increase of $K$. In general, increasing reaction parameter $K$ is to increase the concentration gradient at the surface. The critical point appears to be the same for all values of $K$, which happened because the changes in $K$ does not affect the stretching/shrinking parameter $\lambda$.

Figure 3 displays the velocity profiles $f^{\prime}(\eta)$ for different stretching/shrinking parameter $\lambda$ when $S=3, K=0.5$ and $S c=0.6$. The velocity is shown to decrease with the increase of $\lambda$. We notice that the boundary layer thickness for the second solution is larger than the first solution. It is worth mentioning that the first solutions are stable and physically realizable, while the second solutions are not, as discussed via the stability analysis by Roşca and Pop [15], Weidman et al. [40] and Harris et al. [41]. The profiles displayed in Figure 3 satisfy the far field boundary conditions (10) asymptotically, thus supporting the validity of the dual solutions obtained in this study.

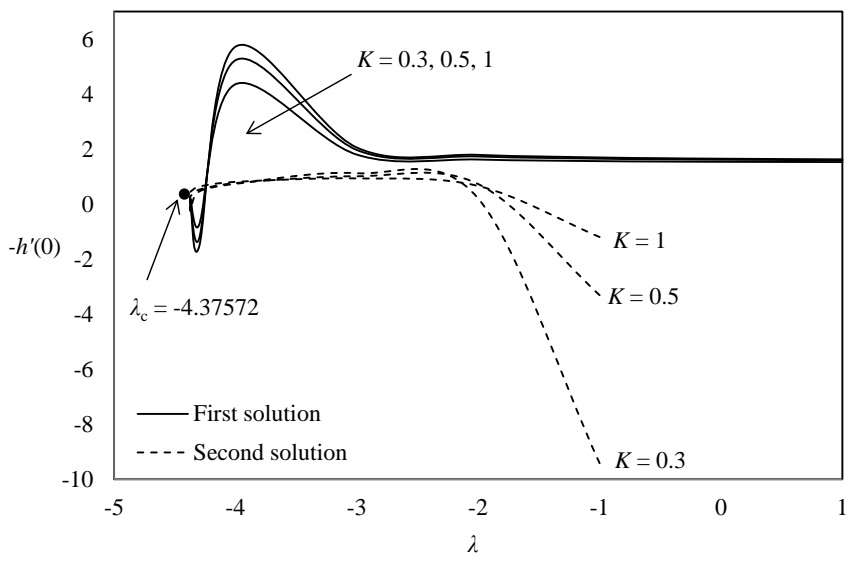

FIGURE II. VARIATIONS OF ${ }^{-h^{\prime}(0)}$ WITH $\lambda$ FOR DIFFERENT K WHEN $S=3, S c=0.6$ 


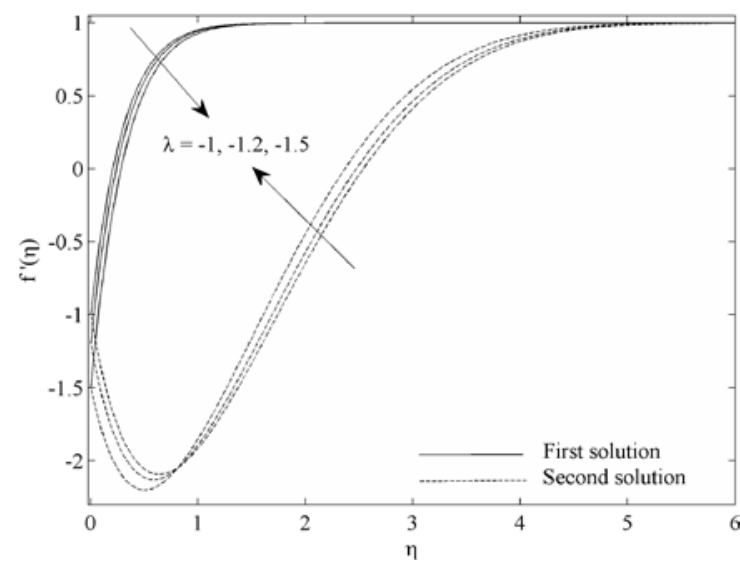

FIGURE III. VELOCITY PROFILES $f^{\prime}(\eta)$ FOR SEVERAL VALUES OF $\lambda_{\text {WHEN }} S=3, K=0.5, S c=0.6$

\section{CONCLUSIONS}

The problem of steady two-dimensional stagnation-point flow and diffusion of chemically reactive species of a viscous and incompressible fluid past a permeable quadratically stretching/shrinking sheet has been studied. This problem is solved numerically by the bvp4c function from MATLAB. The influence of the velocity mass flux parameter $S$, stretching/shrinking parameter $\lambda$, reaction parameter $K$ and Schmidt number Sc have been analyzed and presented graphically. Dual solutions are found for a certain range of the stretching/shrinking parameter. The mass flux parameter $S$ widens the range of $\lambda$ for which similarity solutions exist. The values of the skin friction coefficients increase with the increase of velocity mass flux parameters $S$. The concentration gradient $-h^{\prime}(0)$ decreases with the increase of reaction parameter $K$. The velocity profiles $f^{\prime}(\eta)$ decrease with the increase of the stretching/shrinking parameter $\lambda$.

\section{ACKNOWLEDGMENT}

This work was supported by the following research grants: FRGSTOPDOWN/2014/SG04/UKM/01 from the Ministry of Education, Malaysia and GUP-2013-040 from the Universiti Kebangsaan Malaysia.

\section{REFERENCES}

[1] B. C. Sakiadis, "Boundary-layer behavior on continuous solid surfaces: I. Boundary-layer equations for two-dimensional and axisymmetric flow," AIChE J., vol. 7, pp. 26-28, 1961.

[2] L. J. Crane, "Flow past a stretching plate," Zeitschrift Für Angewandte Mathematik Und Physik, vol. 21(4), pp. 645-647, 1970.

[3] P. S. Gupta and A. S. Gupta, "Heat and mass transfer on a stretching sheet with suction or blowing,” Can. J. Chem. Eng., vol. 55, pp. 744-746, 1977.

[4] K. R. Rajagopal, T. Y. Na and A. S. Gupta, "Flow of a viscoelastic fluid over a stretching sheet,” Rheol. Acta, vol. 23, pp. 213-215, 1984.

[5] H. Xu, I. Pop and X.-C. You, "Flow and heat transfer in a nano-liquid film over an unsteady stretching surface,” Int. J. Heat Mass Transfer, vol. 60, pp. 646-652, 2013.

[6] C.-K. Chen and M.-I. Char, "Heat transfer of a continuous, stretching surface with suction or blowing," J. Math Anal. Appl., vol. 135(2), pp. 568-580, 1988.
[7] E. Magyari and B. Keller, "Exact solutions for self-similar boundarylayer flows induced by permeable stretching walls,” Eur. J. Mech. B. Fluids, vol. 19(1), pp. 109-122, 2000.

[8] M. Miklavcic and C. Y. Wang, "Viscous flow due to a shrinking sheet," Quart. Appl. Math., vol. 64, pp. 283-290, 2006.

[9] M. Sajid, T. Javed and T. Hayat, "MHD rotating flow of a viscous fluid over a shrinking surface,” Nonlinear Dyn., vol. 51(1-2), pp. 259-265, 2008.

[10] T.-G. Fang, J. Zhang and S.-S. Yao, "Viscous Flow over an unsteady shrinking sheet with mass transfer," Chin. Phys. Lett., vol. 26(1), pp. 014703, 2009.

[11] F. Ali, R. Nazar and N. Arifin, "MHD viscous flow and heat transfer induced by a permeable shrinking sheet with prescribed surface heat flux,” WSEAS Trans. on Maths. vol. 9(5), pp. 365-375, 2010.

[12] N. Bachok, A. Ishak and I. Pop, "Unsteady three-dimensional boundary layer flow due to a permeable shrinking sheet,” Appl. Math. Mech., vol. 31(11), pp. 1421-1428, 2010.

[13] K. Bhattacharyya and I. Pop, "MHD boundary layer flow due to an exponentially shrinking sheet," Magnetohydrodynamics, vol. 47(4), pp. 337-344, 2011.

[14] A. M. Rohni, S. Ahmad and I. Pop, "Flow and heat transfer over an unsteady shrinking sheet with suction in nanofluids," Int. J. Heat Mass Transfer, vol. 55(7-8), pp. 1888-1895, 2012.

[15] A. V. Roşca and I. Pop, "Flow and heat transfer over a vertical permeable stretching/shrinking sheet with a second order slip,” Int. J. Heat Mass Transfer, vol. 60, pp. 355-364, 2013.

[16] R. U. Haq, S. Nadeem, Z. H. Khan and T. G. Okedayo, "Convective heat transfer and MHD effects on Casson nanofluid flow over a shrinking sheet,” Cent. Eur. J. Phys., vol. 12(12), pp. 862-871, 2014.

[17] K. Hiemenz, "Die Grenzschicht an einem in den gleichförmigen Flüssigkeitsstrom eingetauchten geraden Kreiszylinder," Dinglers Polytech. J., vol. 326, pp. 321-324, 1911.

[18] F. Homann, "Der Einfluß großer Zähigkeit bei der Strömung um den Zylinder und um die Kugel,” ZAMM, vol. 16(3), pp. 153-164, 1936.

[19] L. Howarth L, "CXLIV. The boundary layer in three dimensional flow.-Part II. The flow near a stagnation point,” Philos. Mag. Ser., vol. 42(335), pp. 1433-1440, 1951.

[20] N. Rott, "Unsteady viscous flow in the vicinity of a stagnation point,". Q Appl. Math., vol. 13, pp. 444-451, 1956.

[21] P. A. Libby, "Wall shear at a three dimensional stagnation point with a moving wall," AIAA J., vol. 12, pp. 408-409, 1974.

[22] J. Iwan and D. Alexander, "Oblique flow onto a growing crystal interface: An exact solution,” J. Cryst. Growth, vol. 89(2-3), pp. 251256, 1988.

[23] P. D. Weidman and S. Mahalingam, "Axisymmetric stagnation-point flow impinging on a transversely oscillating plate with suction,” J. Eng. Math., vol. 31(2-3), pp. 305-318, 1997.

[24] C. Y. Wang, "Axisymmetric stagnation flow towards a moving plate," AIChE. J., vol. 19, pp. 1080-1081, 1973.

[25] C. Y. Wang, “Axisymmetric stagnation flow on a cylinder,” Quart. Appl. Math., vol. 32, pp. 207-213, 1974.

[26] C. Y. Wang, "Similarity stagnation point solutions of the Navier-Stokes equations - review and extension,” Eur. J. Mech. B. Fluids, vol. 27(6), pp. 678-683, 2008.

[27] P. L. Chambré and J . D. Young, "On the diffusion of a chemically reactive species in a laminar boundary layer flow,” Phys. Fluids, vol. 1(1), pp. 48, 1958.

[28] H. I. Andersson, O. R. Hansen and B. Holmedal, "Diffusion of a chemically reactive species from a stretching sheet," Int. J. Heat Mass Transfer, vol. 37(4), pp. 659-664, 1994.

[29] H. S. Takhar, A. J. Chamkha and G. Nath, "Flow and mass transfer on a stretching sheet with a magnetic field and chemically reactive species," Int. J. Eng. Sci., vol. 38(12), pp. 1303-1314, 2000.

[30] A. Raptis and C. Perdikis, "Viscous flow over a non-linearly stretching sheet in the presence of a chemical reaction and magnetic field," Int. J. Non Linear Mech., vol. 41(4), pp. 527-529, 2006. 
[31] I. Muhaimin, R. Kandasamy, I. Hashim and A. Khamis, "On the effect of chemical reaction, heat and mass transfer on nonlinear MHD boundary layer past a porous shrinking sheet with suction,” Theor. Appl. Mech., vol. 36(2), pp. 101-117, 2009.

[32] S. M. M. El-Kabeir, A. Chamkha and A. M. Rashad, "Heat and mass transfer by MHD stagnation-point flow of a power-law fluid towards a stretching surface with radiation, chemical reaction and Soret and Dufour effects," Int. J. Chem. Reactor Eng., vol. 8(1), pp. 1-18, 2010.

[33] Z. Ziabakhsh, G. Domairry, H. Bararnia and H. Babazadeh, “Analytical solution of flow and diffusion of chemically reactive species over a nonlinearly stretching sheet immersed in a porous medium,” J. Taiwan Inst. Chem. Eng., vol. 41(1), pp. 22-28, 2010.

[34] K. Bhattacharyya, "Dual solutions in boundary layer stagnation-point flow and mass transfer with chemical reaction past a stretching/shrinking sheet,” Int. Commun. Heat Mass Transfer, vol. 38(7), pp. 917-922, 2011.

[35] N. C. Roşca, T. Grosan and I. Pop, "Stagnation-point flow and mass transfer with chemical reaction past a permeable stretching/shrinking sheet in a nanofluid," Sains Malaysiana, vol. 41(10), pp. 1271-1279, 2012.

[36] A. Rasekh, M. Farzaneh-Gord, S. R. Varedi and D. D. Ganji, “Analytical solution for magnetohydrodynamic stagnation point flow and heat transfer over a permeable stretching sheet with chemical reaction,” J. Theor. Appl. Mech., vol. 51(3), pp. 675-686, 2013.

[37] M. Abd El-Aziz, "Effect of time-dependent chemical reaction on stagnation point flow and heat transfer over a stretching sheet in a nanofluid,” Phys. Scr., vol. 89(8), pp. 085205, 2014.

[38] N. Najib, N. Bachok, N. M. Arifin and A. Ishak, "Stagnation point flow and mass transfer with chemical reaction past a stretching/shrinking cylinder,” Sci. Rep., vol. 4, pp. 4178, 2014.

[39] J. Kierzenka and L. F. Shampine, "A BVP solver based on residual control and the Maltab PSE,” CM Trans. Math. Softw., vol. 27(3), pp. 299-316, 2001.

[40] P. D. Weidman, D. G. Kubitschek and A. M. J. Davis, "The effect of transpiration on self-similar boundary layer flow over moving surfaces," Int. J. Eng. Sci., vol. 44(11-12), pp. 730-737, 2006.

[41] S. D. Harris, D. B. Ingham and I. Pop, "Mixed convection boundarylayer flow near the stagnation point on a vertical surface in a porous medium: Brinkman model with slip,” Transp. Porous Media, vol. 77(2), pp. 267-285, 2009. 